\title{
Time-dependent global MHD simulations of Cassini T32 flyby: From magnetosphere to magnetosheath
}

\author{
Y. J. Ma, ${ }^{1}$ C. T. Russell, ${ }^{1}$ A. F. Nagy, ${ }^{2}$ G. Toth,${ }^{2}$ C. Bertucci, ${ }^{3}$ M. K. Dougherty, ${ }^{3}$ \\ F. M. Neubauer, ${ }^{4,5}$ A. Wellbrock, ${ }^{6}$ A. J. Coates, ${ }^{6}$ P. Garnier, ${ }^{7}$ J.-E. Wahlund, ${ }^{7}$ \\ T. E. Cravens, ${ }^{8}$ and F. J. Crary ${ }^{9}$
}

Received 13 August 2008; revised 4 December 2008; accepted 19 December 2008; published 7 March 2009.

[1] When the Cassini spacecraft flew by Titan on 13 June 2007, at 13.6 Saturn local time, Titan was directly observed to be outside Saturn's magnetopause. Cassini observations showed dramatic changes of magnetic field orientation as well as other plasma flow parameters during the inbound and outbound segments. In this paper, we study Titan's ionospheric responses to such a sudden change in the upstream plasma conditions using a sophisticated multispecies global MHD model. Simulation results of three different cases (steady state, simple current sheet crossing, and magnetopause crossing) are presented and compared against Cassini Magnetometer, Langmuir Probe, and Cassini Plasma Spectrometer observations. The simulation results provide clear evidence for the existence of a fossil field that was induced in the ionosphere. The main interaction features, as observed by the Cassini spacecraft, are well reproduced by the time-dependent simulation cases. Simulation also reveals how the fossil field was trapped during the interaction and shows the coexistence of two pileup regions with opposite magnetic orientation, as well as the formation of a pair of new Alfven wings and tail disconnection during the magnetopause crossing process.

Citation: Ma, Y. J., et al. (2009), Time-dependent global MHD simulations of Cassini T32 flyby: From magnetosphere to magnetosheath, J. Geophys. Res., 114, A03204, doi:10.1029/2008JA013676.

\section{Introduction}

[2] As Saturn's largest moon, Titan has received particular attention from the ongoing Cassini mission. During the prime mission, Cassini spacecraft made more than 40 Titan passes, providing an extremely valuable database to study Titan's plasma interaction in detail in different regions under varying conditions. Such a data set is also essential for the validation of various numerical models of Titan.

[3] For an unmagnetized body such as Titan, its upper atmosphere/ionosphere is expected to play an important role in the interaction process. At $900 \mathrm{~km}$ and below, Titan's atmosphere is dominated by molecular nitrogen [Smith et al.,

\footnotetext{
${ }^{1}$ Institute of Geophysics and Planetary Physics, University of California Los Angeles, Los Angeles, California, USA.

${ }^{2}$ Department of Atmospheric, Oceanic and Space Sciences, University of Michigan, Ann Arbor, Michigan, USA.

${ }^{3}$ Blackett Laboratory, Space and Atmospheric Physics Group, Imperial College London, London, UK.

${ }^{4}$ Institute of Geophysics and Meteorology, University of Cologne, Cologne, Germany.

${ }^{5}$ Retired.

${ }^{6}$ Mullard Space Science Laboratory, Department of Space and Climate Physics, University College London, London, UK.

${ }^{7}$ Swedish Institute of Space Physics, Uppsala, Sweden.

${ }^{8}$ Department of Physics and Astronomy, University of Kansas, Lawrence, Kansas, USA.

${ }^{9}$ Southwest Research Institute, San Antonio, Texas, USA.

Copyright 2009 by the American Geophysical Union. 0148-0227/09/2008JA013676
}

1982; Waite et al., 2005] with a low percentage of methane, and traces of other hydrocarbons and nitriles. Methane and hydrogen become more and more important at higher altitudes. The exobase, where the mean free path of a particle equals the atmospheric scale height, is estimated to be located between 1400 and $1450 \mathrm{~km}$, on the basis of Ion and Neutral Mass Spectrometer (INMS) observations of the $\mathrm{Ta}, \mathrm{Tb}$, and T5 flybys [De La Haye et al., 2007]. The neutral temperature in Titan's upper atmosphere is nearly $150 \mathrm{~K}$, and the corresponding neutral scale height is about $63 \mathrm{~km}$ near $1000 \mathrm{~km}$ altitude.

[4] The ionosphere of Titan is very complex [Cravens et al., 2006]. Solar extreme ultraviolet radiation and photoelectron impact ionization are the major ionization sources for Titan's dayside ionosphere, while magnetospheric electron impact ionization contributes significantly to the formation of the nightside ionosphere [Keller, 1992; Nagy and Cravens, 1998]. The dominant ion species below $1500 \mathrm{~km}$ are heavy ion species, $\mathrm{C}_{2} \mathrm{H}_{5}^{+}$(mass 28) and $\mathrm{HCNH}^{+}$(mass 29) [Cravens et al., 2006].

[5] Titan's plasma interaction has one further level of complication beyond normal plasma interactions at planets. In the case of planetary interactions, the solar radiation and solar wind flow come from the same direction, while for a moon like Titan, the angle between solar radiation and the corotational plasma flow direction varies from 0 to $180^{\circ}$ as Titan moves along its orbit. The region and amplitude of the plasma flow's perturbation near an unmagnetized body is largely controlled by the intensity of solar radiation (the 
major ionization source), which determines the mass loading rates in the upstream interaction region.

[6] The orbit of Titan is $20 R_{S}$ from Saturn. Under normal solar wind conditions, Saturn's magnetosphere extends from 20 to $25 R_{S}$ toward the Sun [Gombosi and Hansen, 2005]. Several Cassini flybys of Titan (Ta, Tb, T3, T9, and T34) have been studied by various MHD [Backes, 2004; Backes et al., 2005; Ma et al., 2004, 2006, 2007; Neubauer et al., 2006; Snowden et al., 2007] and hybrid models [Kallio et al., 2007; Simon et al., 2008a; Modolo et al., 2007, Modolo and Chanteur, 2008], assuming the upstream plasma to be steady and Titan to be inside Saturn's magnetosphere. The general interaction features include significant slowing down of the plasma flow in the vicinity of Titan and piling up and draping of the magnetic field in the upstream region. While the overall comparison between spacecraft data the models cited above shows relatively good agreement, there are also some significant differences between the calculated and measured parameters, which may be caused by the uncertainties in the upstream conditions and their temporal variations [Ma et al., 2006]. To explain the discrepancy of magnetic field between model results and observations in the deep ionosphere, Neubauer et al. [2006] proposed the idea of a fossil field to describe the fields observed at very low altitudes above Titan. In these regions, the plasma is heavily mass loaded, and thus significantly slowed. Because the field is frozen in with the plasma (except at much lower altitudes where diffusion takes place), the residence time of the field in the ionosphere could be quite long after it gets into Titan's ionosphere. Thus, the field observed at low altitude could have entered Titan's interaction volume one to several hours ago and therefore does not directly relate to the contemporaneous incident ambient field, but to one from a prior time. Simon et al. [2008b] first studied Titan's interaction with changing upstream conditions using a hybrid simulation, but their model failed to reproduce any fossil field.

[7] Although Titan spends most of its time in Saturn's outer magnetosphere, during time of high solar wind pressure, Saturn's magnetopause could move inside Titan's orbit; thus, Titan can be exposed in the magnetosheath region, where the plasma condition is significantly different than the condition in the outer magnetosphere. To estimate the possibility of Titan being outside Saturn's magnetosphere, Gombosi et al. [2006] propagated the solar wind condition measured at Earth radially outward to Saturn. Combining the estimated solar wind pressure with a magnetopause and bow shock model, they found that Titan could spend approximately $20 \%$ of its dayside time in the magnetosheath region and $2 \%$ in the solar wind. Cassini spacecraft caught Titan being outside Saturn's magnetosphere for the first time during the T32 flyby [Bertucci et al., 2008]. In this paper, we use a global MHD model to reproduce the main features observed during this pass by the main plasma instruments on board the spacecraft to explain why they were formed and also to show global interaction structures during this special flyby. The next section discusses the major signatures of the plasma parameters as observed during this flyby. Section 3 briefly introduces the numerical model that is used in the paper. The numerical simulation results of three different cases, as well as comparison between model results and observations, are presented in section 4. Some discussion and summary is given in section 5 .

\section{Observations}

[8] Of the more than 40 Titan passes by Cassini, the T32 flyby is a very special one. During this flyby, as first reported by Bertucci et al. [2008], Cassini spacecraft caught a special moment in which Titan was in a transitional stage, moving from the magnetosphere to the magnetosheath region. The time of the closest approach was 1746 UT on 13 June 2007, at which the spacecraft was only $975 \mathrm{~km}$ altitude above the moon's surface. Titan was located at 13.6 Saturn local time (SLT), close to noon, during the pass. The angle between the ideal corotation flow direction and solar radiation direction was about $60^{\circ}$.

[9] Figure 1 (left) shows the observations made by the Cassini magnetometer [Dougherty et al., 2004] during the flyby. The observed $X, Y$, and $Z$ components (in Titan interaction (TIIS) coordinate system-Titan interaction system) and strength of the magnetic field are plotted along with time. The four vertical lines correspond to the magnetopause crossing (red), closest approach (purple), and two sharp jumps of the field direction in the ionosphere (green), respectively. The red vertical lines correspond to $1731 \mathrm{UT}$, the time that the spacecraft entered the magnetosheath region, after passing through the thick magnetopause layer. The magnetopause crossing took a few minutes [Bertucci et al., 2008], indicating a magnetopause boundary layer about $36,000 \mathrm{~km}$ wide (a 5 min interval). Across the boundary, the magnetic field suddenly reversed its orientation; the $B_{Z}$ component changed from -8 to $10 \mathrm{nT}$. Near the closest approach, as indicated by the purple lines, the magnetic field drops to near zero because of finite magnetic resistivity in the lower ionosphere. The green lines correspond to two local field strength minimums, and the magnetic field sharply rotates near the two corresponding locations, which were in the upper ionosphere of Titan at about 1600 and $1500 \mathrm{~km}$ altitude during inbound and outbound, respectively. These two locations mark roughly the intensified interaction region near Titan.

[10] The trajectory of the T32 flyby is shown in Figure 1 (right), along with the measured magnetic field vectors projected into the equatorial and flow terminator planes. T32 flyby is a north polar pass. The Cassini spacecraft moved in the direction away from Saturn and passed Titan from downstream to upstream, with respect to the ideal corotation flow direction. The magnetopause crossing occurred downstream, about $2.5 R_{T}$ away from Titan, where the magnetic field changed suddenly from southward to northward. The magnetic field stayed northward for nearly 10 min afterward ( 2 blue tick marks), then as the spacecraft approached Titan's ionosphere, the magnetic field vector changed its direction to southward, accompanied by significant disturbance. The field orientation changed to northward again after the spacecraft left Titan's upper ionosphere. Because the field in Saturn's dayside magnetosphere is always pointing southward, it is certain that the region where the field points northward was in the region of Saturn's magnetosheath. This indicates that Titan entered the shocked solar wind with northward field for nearly $15 \mathrm{~min}$ before the closest approach. However, the direction of the field near the 

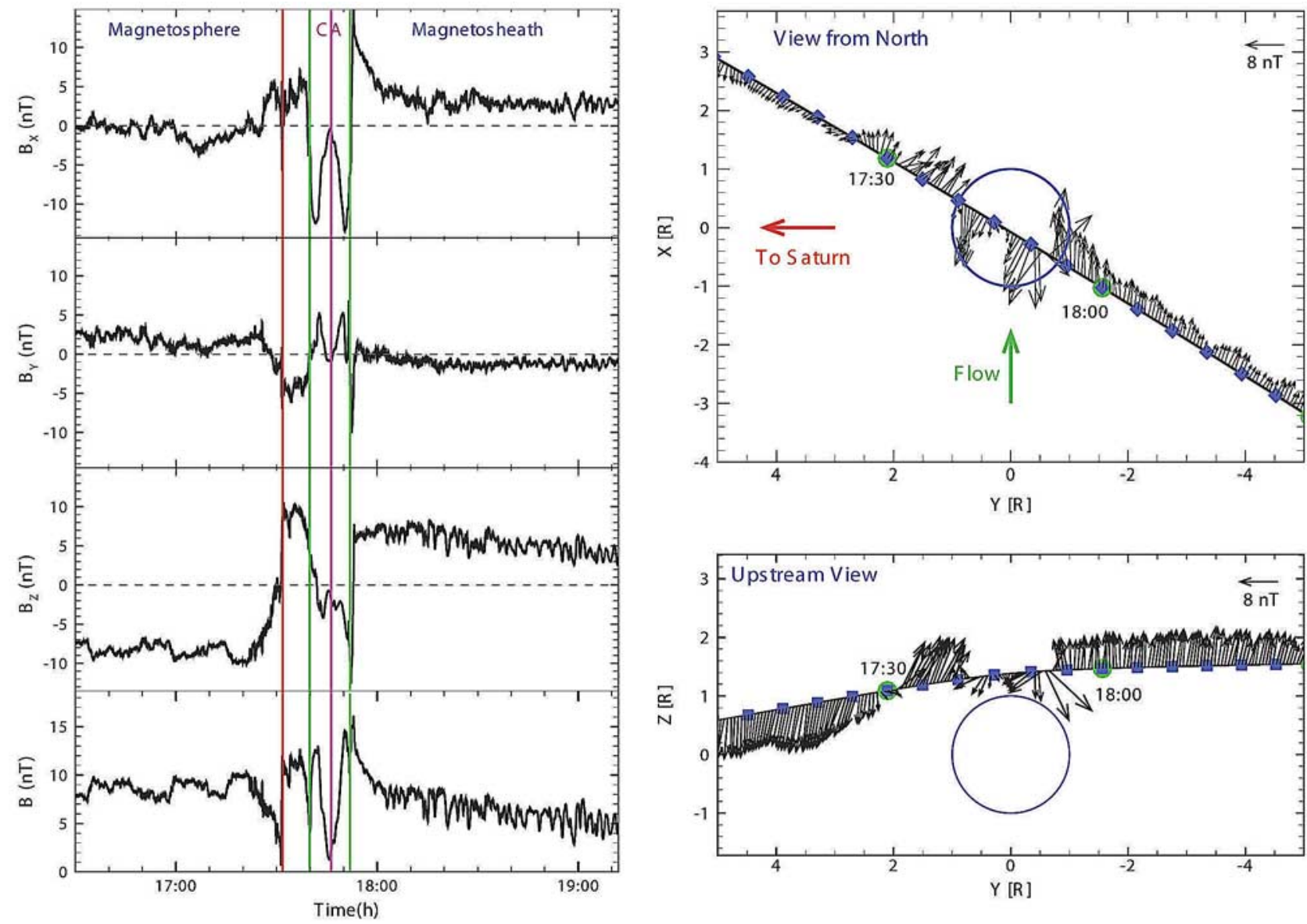

Figure 1. Magnetometer observations during the T32 flyby in TIIS coordinates. (left) Shows the observed $X, Y$, and $Z$ components and strength of the magnetic field along with time. The three vertical lines correspond to magnetopause crossing, closest approach, and the interface in the ionosphere, respectively. (right) Shows the projection of the trajectory of the T32 flyby along with the measured magnetic field vectors projected into the equatorial and flow terminator planes.

North Polar region was opposite to what was expected to be observed, according to a simple draping of the northward field lines around the obstacle. Bertucci et al. [2008] proposes that the southward draping field that was observed near Titan was the fossil field, which was formed because of interaction with Saturn's magnetosphere during an earlier time. The proposed idea explains the observed field orientation. However, how the fossil field was trapped and how it would evolve afterward are still open questions because of the geometrical complexity of the process.

[11] Figure 2 shows measurements of Cassini Plasma Spectrometer (CAPS) [Young et al., 2004] and RPWS Langmuir probe [Gurnett et al., 2004; Wahlund et al., 2005]. The observed values of electron density $\left(n_{e}\right)$, ion velocity $\left(U_{i}\right)$ and electron temperature $\left(T_{e}\right)$ are plotted along with the time, with the same four vertical lines as discussed in Figure 1. Both the electron number density and temperature changed dramatically [Coates et al., 2007] during the pass. The CAPS-ELS geometric factor has recently been recalibrated. Shown in the plot is their latest $1 \mathrm{~s}$ data set of the flyby. As shown in Figure 2 (top), the average $n_{e}$ was about $0.05 \mathrm{~cm}^{-3}$ before the magnetopause crossing, and increased to $0.4 \mathrm{~cm}^{-3}$ during the outbound segment. Although no detailed information on the plasma composition is available,

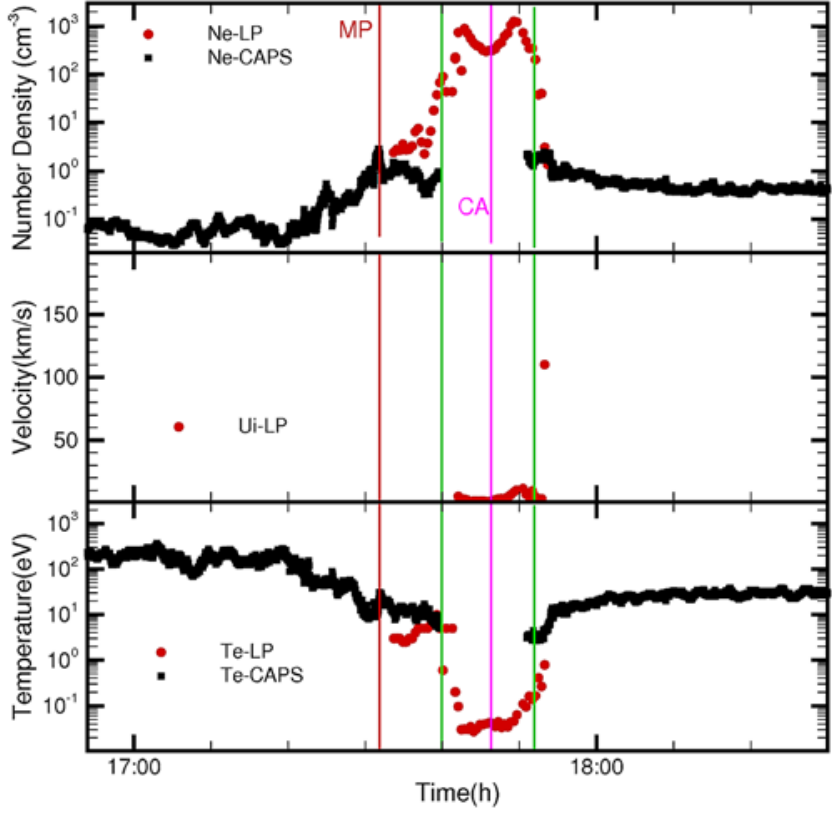

Figure 2. LP and CAPS observations of plasma density, velocity, and temperature during the T32 flyby. 
the plasma before the magnetopause (MP) crossing is expected to contain both protons and heavy ions such as $\mathrm{O}^{+}$, while the magnetosheath plasma during outbound was mostly likely to be dominated by the shocked solar wind protons. Even though the spacecraft passed by mostly nightside ionosphere, the electron density near Titan was still several orders of magnitude higher than the background plasma density, as shown by the Langmuir Probe (LP) data. Also, there is a density dip near the closest approach (CA) with peaks at both sides. The maximum of the electron density reached about $2.0 \times 10^{3} \mathrm{~cm}^{-3}$ outbound at a solar zenith angle (SZA) of nearly $95^{\circ}$. Figure 2 (middle) shows that the ion velocity near Titan, inferred from LP measurements, is significantly lower than the corotating plasma flow speed, as expected in the ionosphere. There is no velocity profile plotted outside the region between the two green lines, because ion parameters in the thin plasma are not reliable when LP reaches the noise level. In addition, during the inbound pass, before 1742 UT, the probe was in eclipse with respect to Titan; as a consequence, no information on the ions was available. Figure 2 (bottom) shows that the electron temperature was $300 \mathrm{eV}$ during inbound before the magnetopause (MP) crossing, and decreased to $20 \mathrm{eV}$ outbound. The observed electron temperature was much lower inside Titan's ionosphere. As indicated by the LP data, the temperature was about $0.04 \mathrm{eV}(\sim 400 \mathrm{~K})$ near the closest approach (CA).

\section{Model Description}

[12] We use the 7 species single-fluid MHD model, as described by $M a$ et al. [2006]. The equations are solved using the BATSRUS code [Powell et al., 1999]. The model considers 7 pseudoion species: $\mathrm{L}^{+}$(mass 1), $\mathrm{M}^{+}$(mass 14), $\mathrm{C}_{2} \mathrm{H}_{5}^{+}(\operatorname{mass} 29), \mathrm{HCNH}^{+}(\operatorname{mass} 28), \mathrm{MHC}^{+}(\operatorname{mass} 44)$, $\mathrm{HHC}^{+}$(mass 70), and $\mathrm{HNI}^{+}$(mass 74). The calculation of the mass densities of each ion species takes into account the major chemical reactions including photoionization, impact ionization, charge exchange and recombination selfconsistently. All the ion species share the same velocity and temperature. The combined momentum and energy equations consider the effects of both elastic and inelastic ion neutral collisions. Ten background neutrals $\left(\mathrm{N}_{2}, \mathrm{CH}_{4}, \mathrm{H}\left(\mathrm{H}_{2}\right), \mathrm{C}_{2} \mathrm{H}_{2}\right.$, $\mathrm{C}_{2} \mathrm{H}_{4}, \mathrm{C}_{2} \mathrm{H}_{6}, \mathrm{C}_{3} \mathrm{H}_{4}, \mathrm{C}_{4} \mathrm{H}_{2}, \mathrm{HCN}$, and $\mathrm{HC}_{3} \mathrm{~N}$ ) are included in the model. The photoionization rates used are dependent on the SZA with optical depth considered [Cravens et al., 2006].

[13] The inner boundary is set at $725 \mathrm{~km}$, where the collisions are dominant. We assume photochemical equilibrium for the ionospheric ion species. The plasma temperature is set to twice the neutral temperature $\left(T_{P}=2 T_{N}=300\right.$ $\mathrm{K}=0.3 \mathrm{eV})$. An absorbing boundary has been used for $u$ and $B$, which enforces a zero gradient in velocity and magnetic field vectors across the boundary. This boundary is consistent with conditions near $725 \mathrm{~km}$, because the strong ion neutral collision results in nearly zero velocity, and the strong magnetic diffusion coefficient results in nearly zero gradient of the magnetic field. The boundary condition in our model is applied with the help of ghost cells that are located inside the inner boundary. The state variables (such as density, velocity, magnetic field and temperature) of the plasma in these ghost cells can provide a first-order accurate gradient for state variables of the adjacent real cell when needed. The zero- gradient boundary condition is applied by setting the values in the ghost cells equal to those of the adjacent real cells, which are calculated on the basis of the physical equations. Such a boundary condition does not enforce the magnetic field to be zero across the boundary, thus allowing the field to diffuse through the interior of the moon.

[14] A spherical grid structure is used in the model inside the computational domain, as defined as $-24 R_{T} \leq$ $X \leq 48 R_{T},-32 R_{T} \leq Y, Z \leq 32 R_{T}$ in TIIS coordinates. The radial resolution ranges from $57 \mathrm{~km}$ near the ionosphere to $3000 \mathrm{~km}\left(\sim 1.15 R_{T}\right)$ near the outer boundary, and angular resolution is $5^{\circ}$. (Note that for the steady state case, the radial resolution is $28 \mathrm{~km}$ with $2.5^{\circ}$ angular resolution below $2000 \mathrm{~km}$ altitude, and the resolution in the ionospheric region is decreased for time-dependent simulation so that it can be finished in a reasonable amount of time). Also, the Hall effect was neglected in the calculation to speed up the runs. The simulations shown in the paper were run by the NASA Columbia supercomputer. A typical time-dependent case requires 1500 processor hours with each time step corresponding to $0.1 \mathrm{~s}$ in physical time. In comparison, a high-resolution steady state simulation needs 1000 processor hours with a local time stepping scheme (using varying time steps in different regions).

\section{Simulation Results}

[15] To understand the plasma observations of the T32 flyby, we present numerical results of three different cases using the global MHD model described above. Since there is no flow information available, we chose the flow velocity to be $U=(120,0,0) \mathrm{km} / \mathrm{s}$ in the calculation. The plasma could be moving at a different speed or in a different direction, especially when Titan is in the magnetosheath. Such differences should not affect the main conclusion of the paper. The plasma flow in the sheath region was submagnetosonic since no bow shock was observed in the upstream region of Titan during the outbound of the flyby. In addition, this flyby happened in the afternoon sector, so the shocked solar wind diverted around the obstacle in a direction somewhat aligned with the corotating plasma flow inside the magnetosphere, making the case simpler. But in general the direction of the flow can be very different from sheath to magnetosphere, in which case, the plasma wake would also change direction significantly on crossing the magnetopause, especially when the crossing happens in the morning sector.

\subsection{Case 1: Steady State}

[16] We first run a steady state case with Titan located in the magnetosphere to see what the spacecraft would have observed if Titan did not encounter with the magnetopause. We use the plasma parameters of Saturn's nominal magnetosphere condition. Specifically, the other parameters for the steady state case are chosen as: $B=(0,0,-8) \mathrm{nT}$, $n_{e}=0.2 \mathrm{~cm}^{-3}$ (with $n\left(\mathrm{~L}^{+}\right)=0.1 \mathrm{~cm}^{-3}, n\left(\mathrm{M}^{+}\right)=0.1 \mathrm{~cm}^{-3}$ ) and plasma temperature $T_{P}=1500 \mathrm{eV}$. The above set of parameters corresponds to a subsonic $\left(V_{S}=146 \mathrm{~km} / \mathrm{s}\right)$ and sub-Alfvenic $\left(V_{A}=143 \mathrm{~km} / \mathrm{s}\right)$ plasma flow, with a fast magnetosonic Mach number of 0.6. The plasma flow is sub-Alfvenic during the T32 flyby since the magnetic field is stronger $(8 \mathrm{nT})$ than that of most other Titan flybys. The 

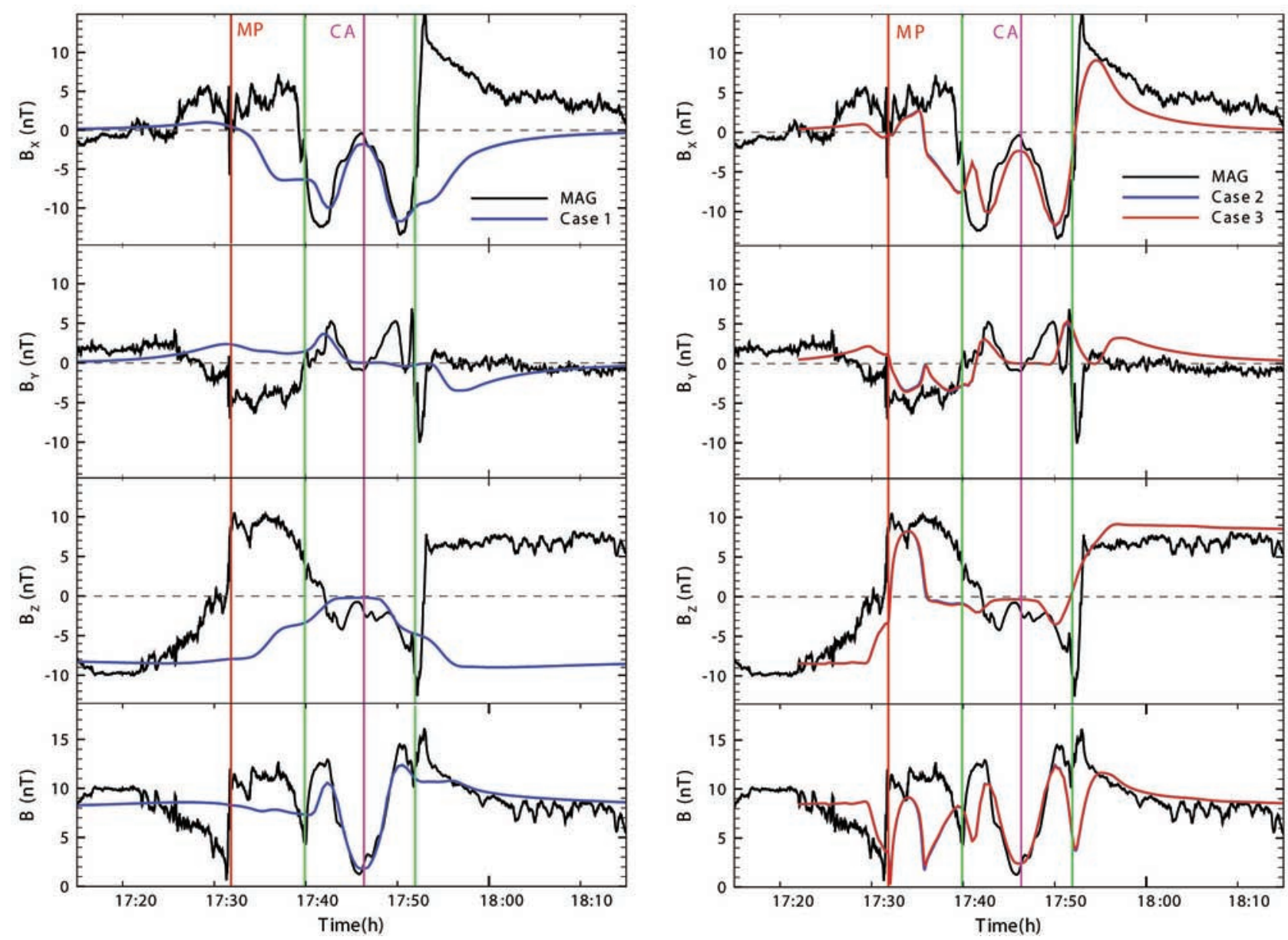

Figure 3. Model and data comparison of the magnetic field along the T32 trajectory. The black lines are MAG observations, and the blue lines are model results. (left) Data comparison with steady state case (case 1) is shown; (right) comparison with current sheet crossing (red) and magnetopause crossing (case 3 ) are plotted. Note the results of case 2 and case 3 overlap each other.

general interaction structures are similar for sub-Alfvenic and super-Alfvenic flow except that with super-Alfvenic flow, the current sheet is thinner and the lobes are closer, while the lobes are separated more from each other in the sub-Alfvenic cases [Ledvina et al., 2004].

[17] The simulation results along the trajectory are examined and compared with plasma observations. Figure 3 (left) shows model and data comparisons of the magnetic field along the T32 trajectory of this case. The black lines represent Cassini Magnetometer (MAG) observations, and the blue lines denote model results. There is good agreement between the calculated and observed values between the two green lines for the $B_{X}$ component, which is also the main magnetic component. This provides clear evidence that the measured magnetic field in the region close to Titan is a fossil field that was formed when Titan was inside the magnetosphere. The discrepancy of the $B_{Y}$ and $B_{Z}$ components could be due to small perturbations of the magnetic field which are neglected in the calculation. Also, both model result and observation show a clear drop of the magnetic field strength near the closest approach. The decreasing starts at approximately $1200 \mathrm{~km}$ altitude. Below this altitude, the plasma is only partially ionized, and magnetic diffusion is important. Thus, in the lower ionosphere of Titan, plasma is no longer frozen to the magnetic field. The decrease of the field can only be reproduced by the model when the magnetic diffusion term is included in the induction equation. It is also important to note that the magnetic resistivity in our calculation is determined by both neutral number densities and electron density [Ma et al., 2006].

[18] Case 1 of Figure 4 shows the comparison of other plasma parameters along the trajectory. Near closest approach, the calculated electron number density profile has two peaks as observed. Also similar to observation, the predicted peak electron density outbound is higher than that inbound. The calculated highest density is $2.5 \times 10^{3} \mathrm{~cm}^{-3}$, slightly larger than that observed by the Langmuir Probe (LP). The calculated velocity between the two green lines is very low, similar to values as estimated on the basis of the LP data, indicating intensified mass loading of the plasma and thus significant slow down of the plasma flow. Since our MHD model only calculated the plasma temperature (sum of electron and ion temperatures), we overplotted in Figure 4 (bottom) with calculated $T_{P} / 2$ (half of the plasma temperature), as an approximation of the electron temperature, assuming that $T_{e}=T_{i}=T_{P} / 2$. The model temperature has a similar trend of variation compared with observations. It is lower than the observation inside the two green lines and 


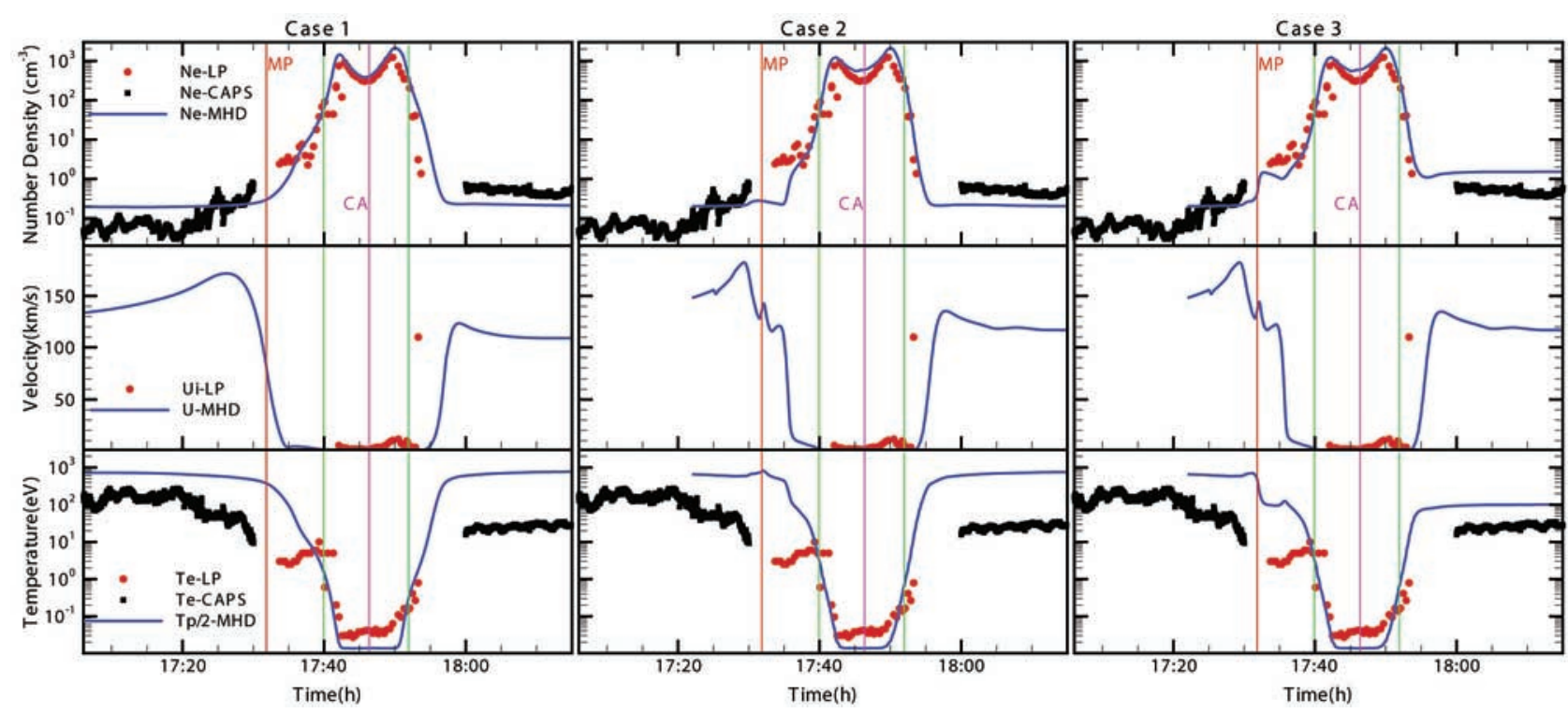

Figure 4. Model and data comparison of plasma density, velocity, and temperature along the trajectory for the three cases.

higher than the observation outside the region, because the electron temperature is higher than ion temperature in the ionosphere and lower than the ion temperature in the magnetospheric plasma flow.

\subsection{Case 2: Current Sheet Crossing $\left(B_{Z}=-8 \mathrm{nT} \rightarrow B_{Z}=8 \mathrm{nT}\right)$}

[19] The good agreement between model results of the first case and observations near Titan clearly demonstrates that the magnetic field observed near Titan is fossil field. The purpose of this case is to explain how the fossil field was trapped inside the ionosphere by simulating Titan's time-dependent interaction with a simple current sheet representing the magnetopause crossing. In this case, the magnetopause boundary layer is simplified to a thin current sheet with $\mathrm{B}_{\mathrm{Z}}$ changed from -8 to $+8 \mathrm{nT}$ (tangential discontinuity). The other parameters are kept the same as in case 1 . We first run the simulation with plasma parameters of case 1 . After it reaches the steady state $(\sim 2 \mathrm{~h}$ physical time), the current sheet begins to enter the simulation domain from upstream $\left(X=-24 R_{T}\right)$ at $1722 \mathrm{UT}$. The thickness of the current sheet corresponds to the grid resolution near the upstream outer boundary, which is about $1000 \mathrm{~km}$. According to the plasma flow speed, the current sheet should arrive at Titan in about $8 \mathrm{~min}$ and $35 \mathrm{~s}(d t=24$ $R_{T} / 120 \mathrm{~km} / \mathrm{s}=515 \mathrm{~s}$ ), which is the same time that the magnetopause crossing is observed.

[20] The fly through of the time-dependent simulation results is plotted in Figure 3 (right) along with the magnetometer observations. The main features of the observed magnetic field are well reproduced by the case. As shown by the blue line, the sharp change of the $B_{Z}$ component at the magnetopause happens at the same time with comparable magnitude as shown by the observations. After that the northward $B_{Z}$ in the simulation only lasts for $4 \mathrm{~min}$, while the observations show that it stayed for about $8 \mathrm{~min}$. The discrepancy is most likely due to the flow direction of the magnetosheath plasma being different from the ideal coro- tation direction, as assumed in the simulation. The real thickness/velocity of the magnetopause could also contribute to the discrepancy. The predicted magnetic field between the two green lines is nearly the same as case 1 , and agrees well with the observation. There is a short delay of the predicted entry of the fossil field region during inbound, but the location of the interface between the old field and the new field during the outbound passage is rather well reproduced. Near the second green line, both model and observation show that $B_{X}$ and $B_{Z}$ components change suddenly from negative to positive values.

[21] Case 2 of Figure 4 shows the comparison of electron densities and temperatures along the trajectory for the current sheet crossing case. Again, between the two green lines, the electron number densities are well reproduced. Because of the lower resolution used in the time-dependent simulation, the agreement near CA is not as good as case 1, but there is a similar trend, indicating that the lower resolution in the ionosphere did not substantially alter the results. The density drop is sharper in this case and thus fits better with the observation outside the ionosphere. There is also a small density disturbance across the magnetopause as compared with case 1 . The predicted plasma flow speed and temperature are very similar to case 1 except for some small disturbances near the magnetopause crossing.

[22] This simple current sheet crossing case reproduces the main features as observed by the magnetometer. To explain how the fossil field was trapped, we plot magnetic field strength and directions in the $X Z$ plane at four different times in Figure 5. The white lines are $B_{Z}=0$ contour lines, as an indication of the interface between the old field (magnetospheric field $B_{Z}<0$ ) and new field (magnetosheath field $B_{Z}>0$ ). The interface was a straight plane parallel to $Y Z$ plane when it entered the simulation domain in the far upstream region. It convected together with the collisionless plasma flow. At 1730:00 UT, the interface arrived right in front of Titan. The interface was also slightly distorted, with the center region lagging behind because of the slow down 

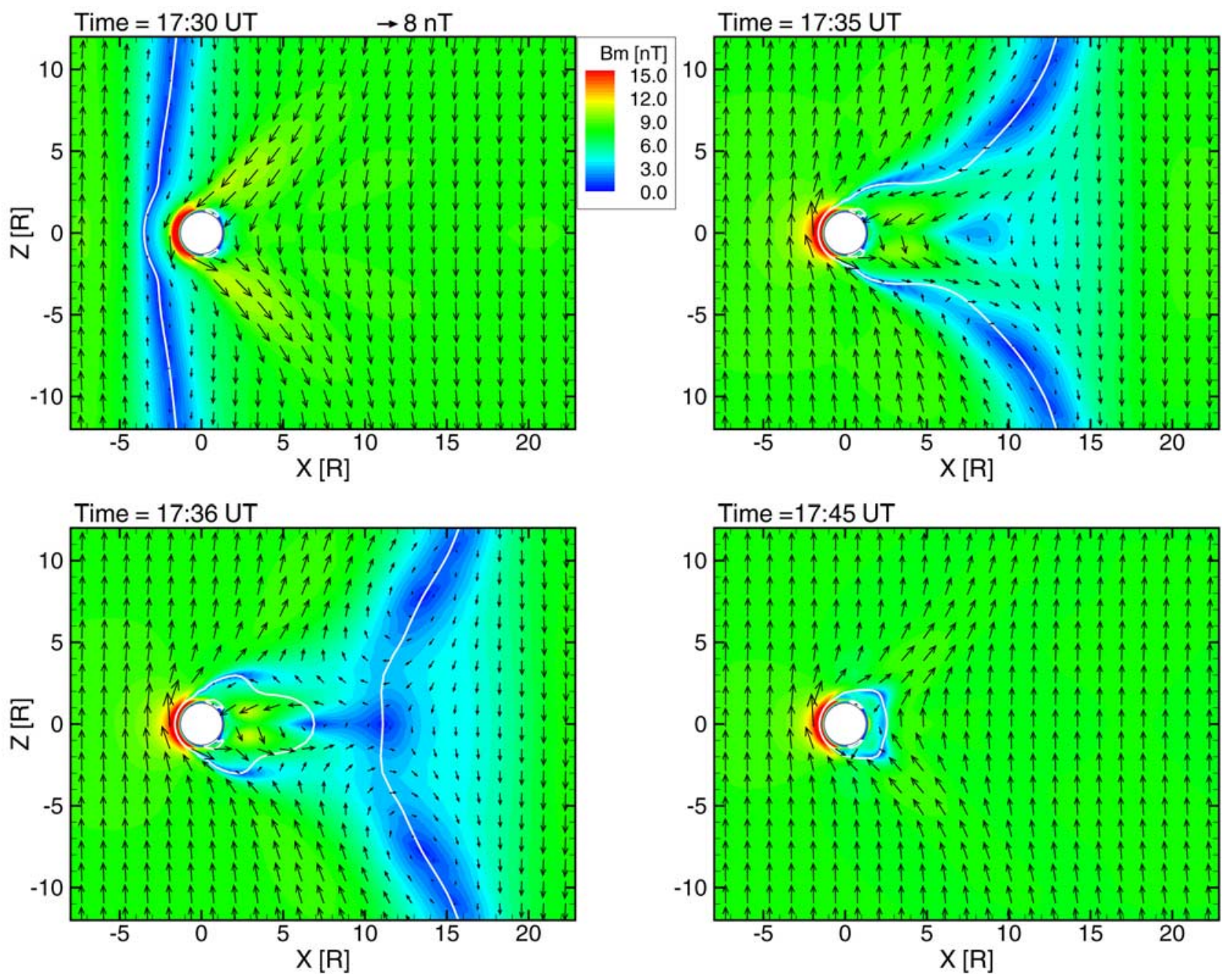

Figure 5. Contour plots of the magnetic field strength at four different times. The arrows show the direction of the magnetic field. The black lines are $B_{Z}=0$ contour lines, as an indication of the interface between the old field $\left(B_{Z}<0\right)$ and new field $\left(B_{Z}>0\right)$.

of the plasma flow. The distortion becomes more and more severe with time. In front of Titan, the northward field gradually piles up above the old southward pileup region, which is also significantly compressed. At 1735:00 UT, the interface reaches to about $X=10 R_{T}$ behind Titan at outside flank region, while the center of the interface is still in front of Titan. The interface finally broke up into two pieces far downstream at 1736 UT. The straight piece moved away from Titan quickly, while the other one formed a closed region near Titan. Inside the closed region, the magnetic field is the fossil field with the same field orientation as in the plasma inside Saturn's magnetosphere, and this region slowly shrinks. The complete erosion of the old field took nearly two hours in the simulation. This is in agreement with the timescales proposed by Bertucci et al. [2008].

[23] The breakup of the interface is due to the slippage of the field lines around the moon in the plane perpendicular to the magnetic field. Magnetic field lines can be stretched to a great distance in the plane containing the field direction. However, because of the 3-D nature of the interaction, the downstream region, except for the current sheet, was soon filled with magnetosheath plasma with northward magnetic field lines slipping around the obstacle as shown in Figure 6. Figure 6 shows the snapshot of $B_{Z}$ component in $X Y$ plane at the same four times. The plasma in the blue color region is associated with the old magnetospheric field. At $1730 \mathrm{UT}$, the interface (black line) was only slightly distorted. Five minutes later, the plasma with southward pointed magnetic field is significantly compressed by the magnetosheath plasma in the distant tail region. The thin tail was shortly broken at $\sim 8 R_{T}$ away from Titan. The enclosed region thus has a complicated 3-D structure, being highly compressed in the $Y$ direction.

[24] Figure 7 shows plasma flow speed and direction at the same four different times. The convection of the interface is mainly controlled by plasma flow speed. The shape of the interface is distorted where the flow was significantly slowed by the obstacle. The plasma flow was disturbed ahead of the interface through fast magnetosonic waves. A pair of new Alfven wings forms in front of the old Alfven wings as the interface passes by Titan. At 1735 UT, the contour plot clearly shows the coexistence of two pairs of Alfven wings. The new wing gradually grows while the old wing shrinks and moves tailward along with the time. 

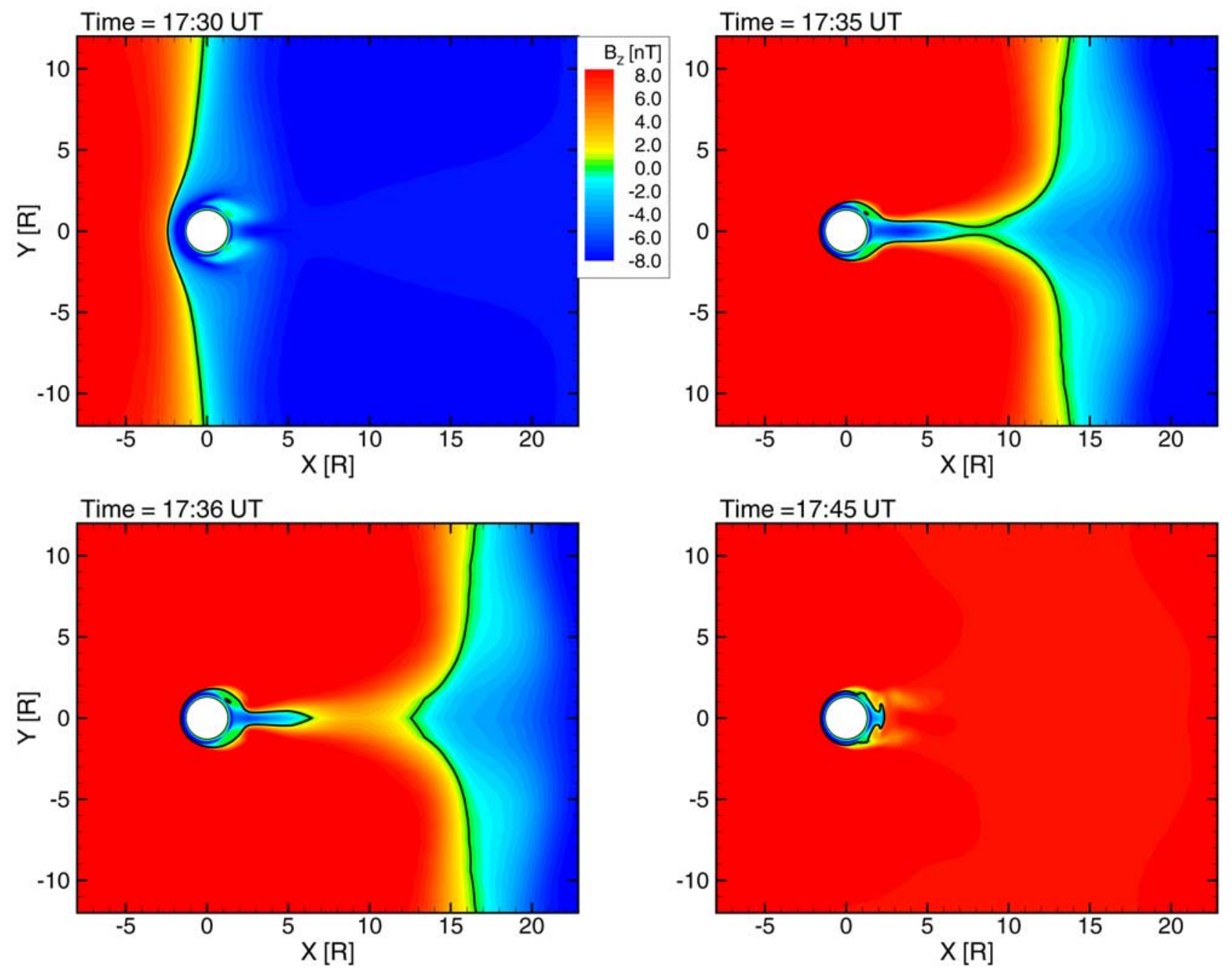

Figure 6. Contour plots of the $B_{Z}$ component in the $X Y$ plane at four different times. The black lines are are $B_{Z}=0$ contour lines, as an indication of the interface between the old field $\left(B_{Z}<0\right)$ and new field $\left(B_{Z}>0\right)$.

The old wings are detached from the moon a few minutes later (not shown). At $1745 \mathrm{UT}$, the distortion of the flow is restored, the old wings completely disappear, and only the new pair of wings remains.

[25] When the oppositely directed magnetic field reaches the obstacle, reconnection occurs on the upstream side when the plasma is significantly slowed down. Because the plasma flow is sub-Alfvenic, the Machcone angle of the Alfven wave is slightly larger than $45^{\circ}$ and the reconnected magnetic flux cannot escape through the tail region efficiently. As a result, magnetic islands form at the flank region, as shown in Figure 8 (left). Figure 8 (right) shows that plasma flow is accelerated in two very limited regions near the upstream reconnection site.

[26] The tail disruption process at Titan is different than a tail disconnection event of a comet in both reconnection efficiency and timescales (For examples, see a comet disconnection event as produced by Jia et al. [2008] with a MHD model). These differences are caused by the following:

[27] 1. Different upstream plasma conditions. The solar wind plasma is fast magnetosonic flow, while the plasma near Titan is usually subsonic and trans-Alfvenic. In other words, dynamic pressure is the dominant pressure for solar wind plasma, so the field lines are draped significantly and results in a very thin current sheet in the comet tail, and the reconnected magnetic flux can escape through the tail region easily. While in the case of Titan, the thermal pressure is the main pressure of the plasma in the upstream region; the angle of the draping field line around the obstacle is determined by the angle of the Alfven wings.

[28] 2. Different neutral envelopes around the two objects. The scale height of the neutrals is much smaller in the case of Titan, so the main interaction region is close to Titan. The tail disruption happens in timescales of $10 \mathrm{~min}$. While in the case of a comet, the neutral cloud extends to very far distance from the nucleus, so a comet has a much wider mass-loading region, the tail disconnection happens in timescales of at least several hours.

[29] Although we are solving the resistive MHD equations, the magnetic resistivity $(\eta)$ near the upstream reconnection site is in the order of $0.1-1.0 \mathrm{~km}^{2} / \mathrm{s}$, so the magnetic diffusion timescale is $\Delta x^{2} / \eta$, corresponding to $1-10 \mathrm{~h}$ when taking the typical length to be the same as the grid size in that region. The magnetic reconnection is triggered by 

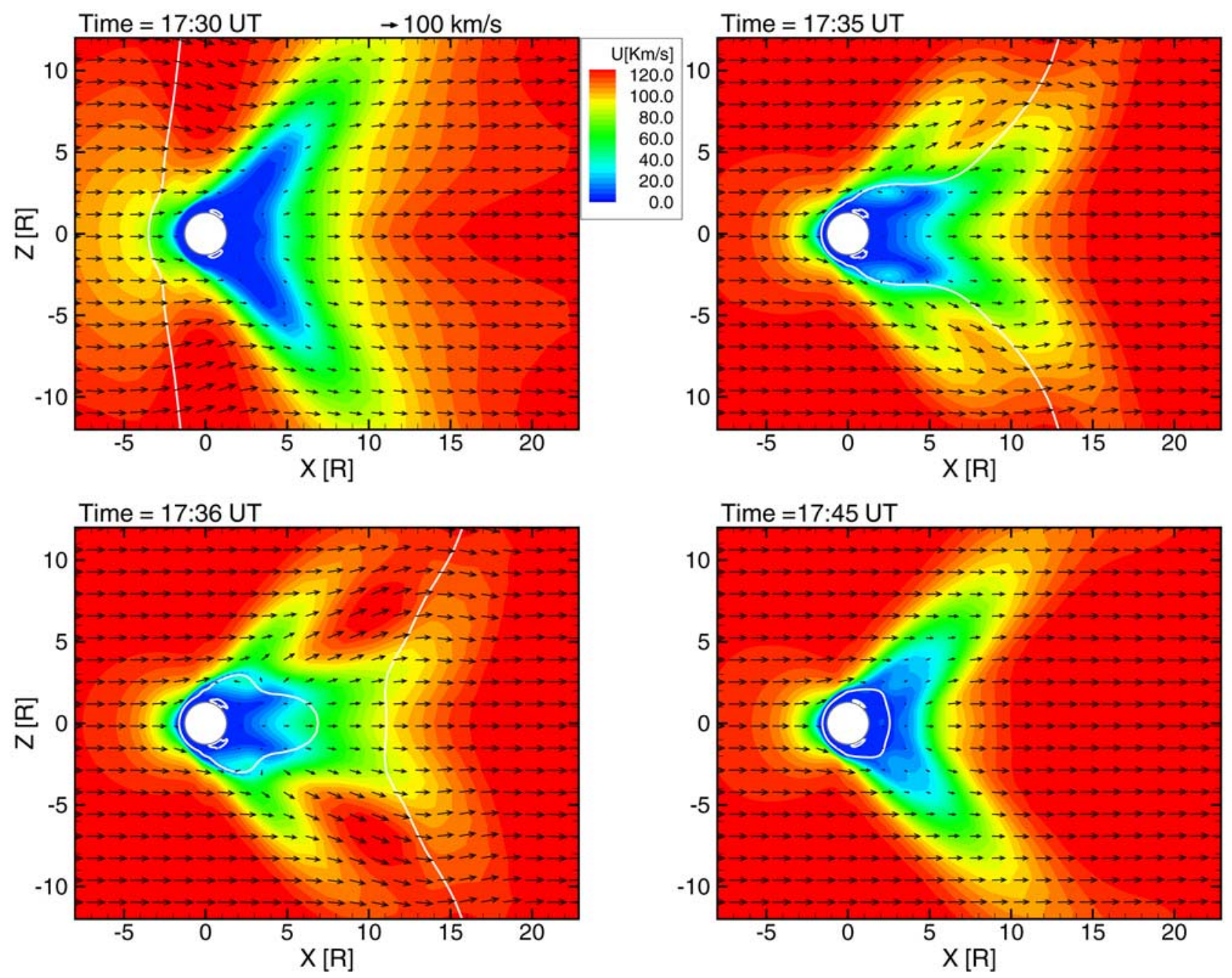

Figure 7. Contour plots of plasma speed at four different times. The arrows show the direction of the plasma flow. The black lines are $B_{Z}=0$ contour lines, as an indication of the interface between the old field $\left(B_{Z}<0\right)$ and new field $\left(B_{Z}>0\right)$.
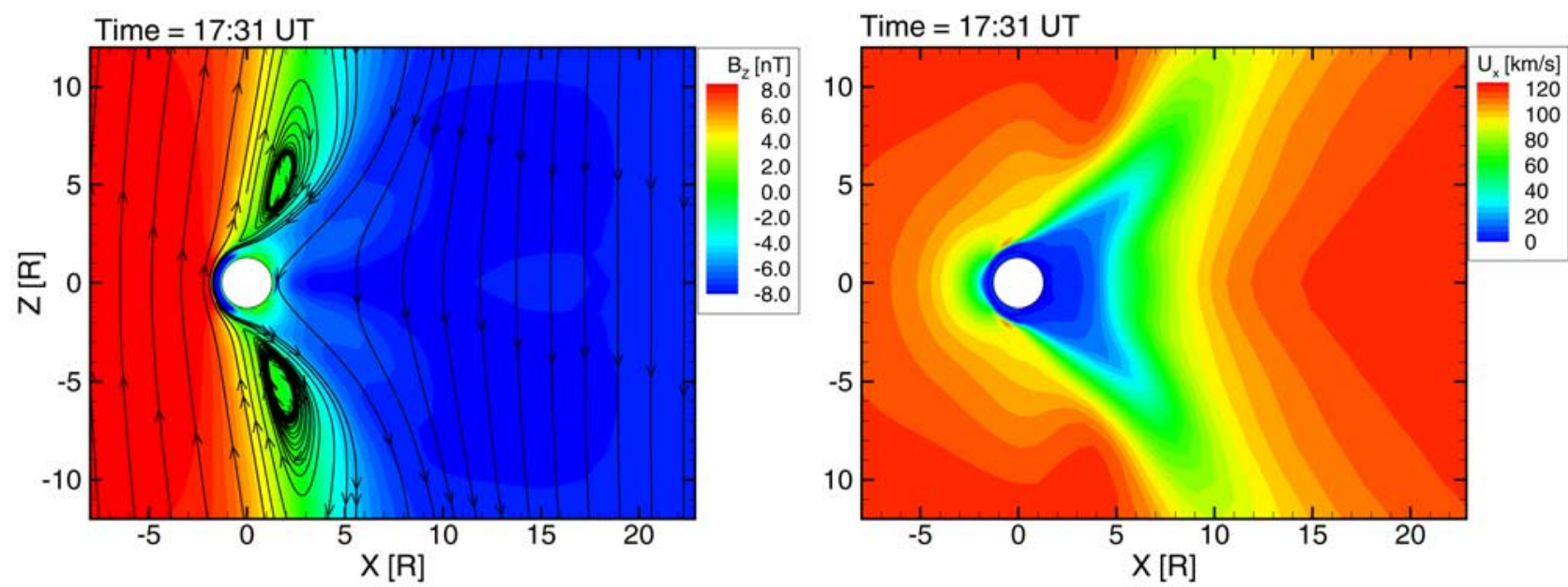

Figure 8. Coutour plot of $B_{Z}$ and $U_{X}$ at 1731 UT in the $X Z$ plane. The black lines are magnetic field lines. 

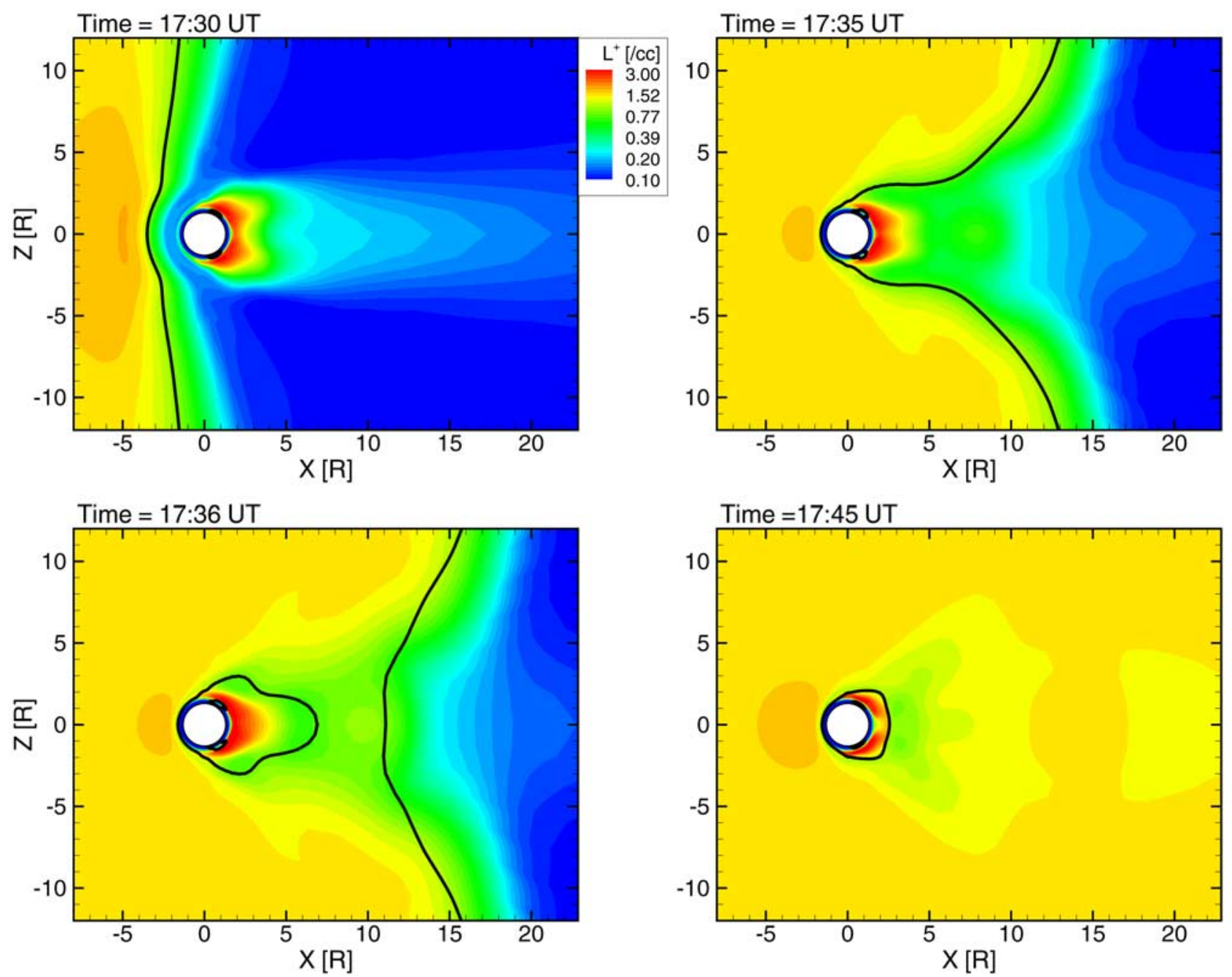

Figure 9. Contour plots of the $\mathrm{H}^{+}$number density at four different times. The purple lines are $B_{Z}=0$ contour lines, as an indication of the interface between the old field $\left(B_{Z}<0\right)$ and new field $\left(B_{Z}>0\right)$.

numerical diffusion, since the magnetic diffusion time is much longer than the reconnection timescale produced by the model. Strictly speaking, the MHD model does not apply to the distant tail region of Titan because of the large gyroradii of the heavy ion species [Ma et al., 2007]. Even though we are confident about the general interaction structure as produced by the model, the detailed tail disruption process could be somewhat different.

\subsection{Case 3: Magnetopause Crossing}

[30] Case 2 does not take into account the enhancement of the electron density and the decrease of the temperature as observed by CAPS data. In case 3 , we also change electron number density and temperature to $n_{e}=1.5 \mathrm{~cm}^{-3}$ (with $n\left(\mathrm{~L}^{+}\right)=1.5 \mathrm{~cm}^{-3}, n\left(\mathrm{M}^{+}\right)=0.0$ ), and $T_{P}=200 \mathrm{eV}$, respectively, at the upstream boundary along with the magnetic field orientation changes, as described in case 2 . In this case, the magnetosheath plasma is assumed with only light species $\left(\mathrm{L}^{+}\right)$, compressed from the solar wind. Even though the number density and plasma temperature change across the boundary, plasma mass density and pressure still maintain equilibrium across the current sheet.
[31] Simulation results of the magnetic field are plotted in red in Figure 3 (right). Cases 2 and 3 give almost identical results (the red and blue curves "overlap," and thus, cannot be distinguished) along the trajectory, indicating that as long as the mass density and pressure are balanced, magnetic perturbations are not sensitive to number density or temperature changes. Even a simple current sheet crossing could reproduce the major signatures of the observed magnetic field.

[32] Case 3 of Figure 4 shows the comparison of electron densities and temperatures along the trajectory. Again, between the two green lines, the electron number densities are well reproduced as in the other two cases. There is a clear increase of the calculated density cross the MP which matches better with the LP density. The plasma flow speed is the same as in case 2. The temperature also matches better with the CAPS observation, especially across the MP and during outbound.

[33] Figure 9 shows contour plots of the proton number density at the same four different times. At 1730 UT, the magnetosheath plasma, which contains relative high-density protons as shown in yellow, has just arrived at Titan. The 

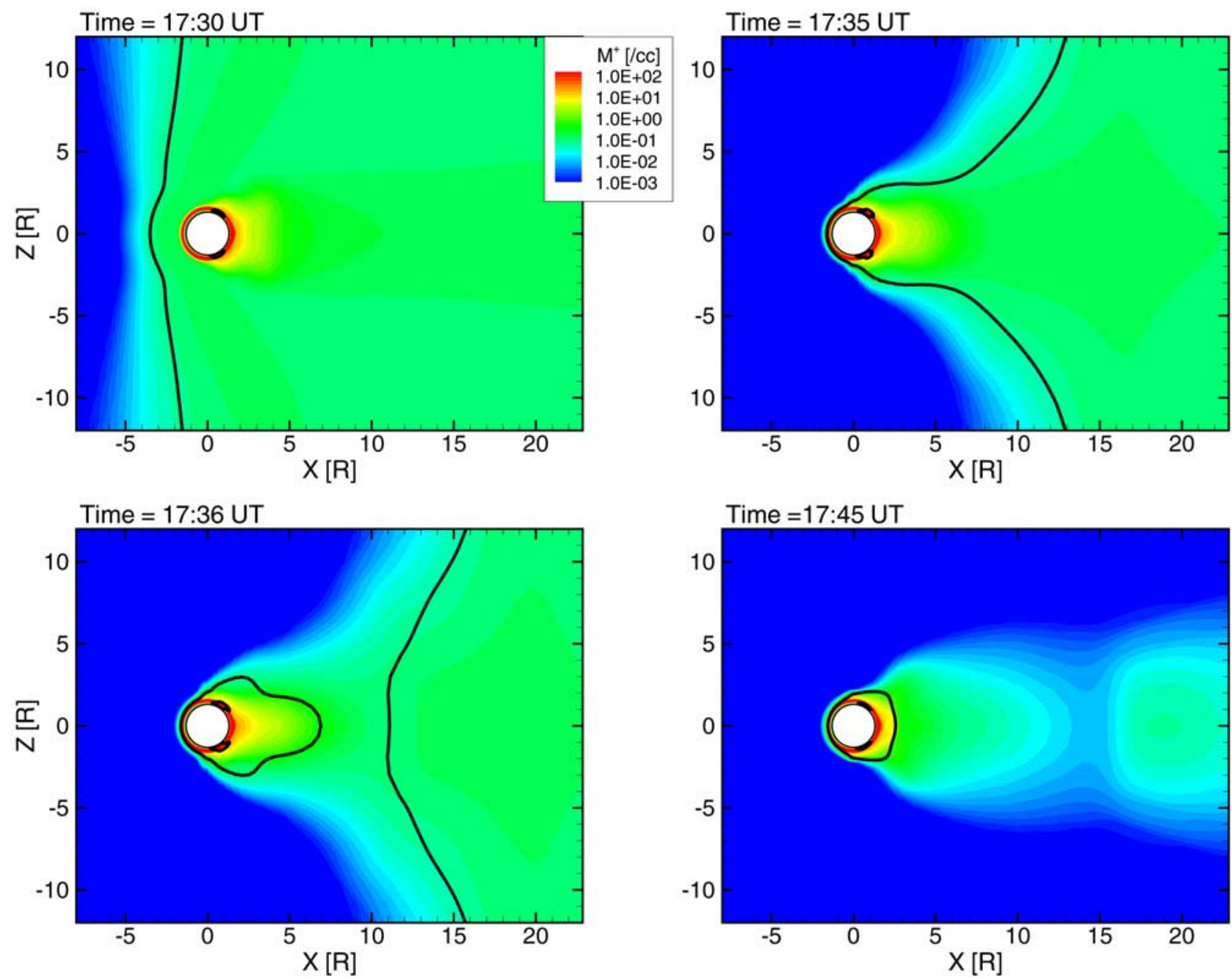

Figure 10. Contour plots of the $\mathrm{M}^{+}$number density at four different times. The purple lines are $B_{Z}=0$ contour lines, as an indication of the interface between the old field $\left(B_{Z}<0\right)$ and new field $\left(B_{Z}>0\right)$.

outside region was quickly filled with magnetosheath plasma in a few minutes along with the interface. At 1736 UT, only the wake region still contained the plasma with low proton density. The highest proton density, as indicated in red, was only a few $\mathrm{cm}^{-3}$, and it is accumulated in the small region around Titan downstream.

[34] Figure 10 shows contour plots of the $\mathrm{M}^{+}$number density at four different times. $\mathrm{M}^{+}$is one of the upstream plasma flow components for the magnetospheric plasma but not in the magnetosheath plasma. So the $\mathrm{M}^{+}$in the outside region was pushed away by the sheath plasma shortly after the interface arrived at Titan. Also, $\mathrm{M}^{+}$is not the major ion in the Titan ionosphere. The peak ion density is only $500 \mathrm{~cm}^{-3}$. There is a tail disconnection associated with the breakup of the interface at 1736 UT. About 4 min later, another tail disconnection happens in the near tail region $\sim 4 R_{T}$, then the plasmoid moves downstream and reaches nearly $15 R_{T}$ at 1745 UT. The plasmoid associated with the first disconnection only contains $\mathrm{M}^{+}$, while for the second breakup, the plasmoid contains also the main ionosphere ion species $\mathrm{C}_{2} \mathrm{H}_{5}^{+}$, suggesting the origin of the newly formed plasmoid. During the later breakup, the escape rate of the heavy ions

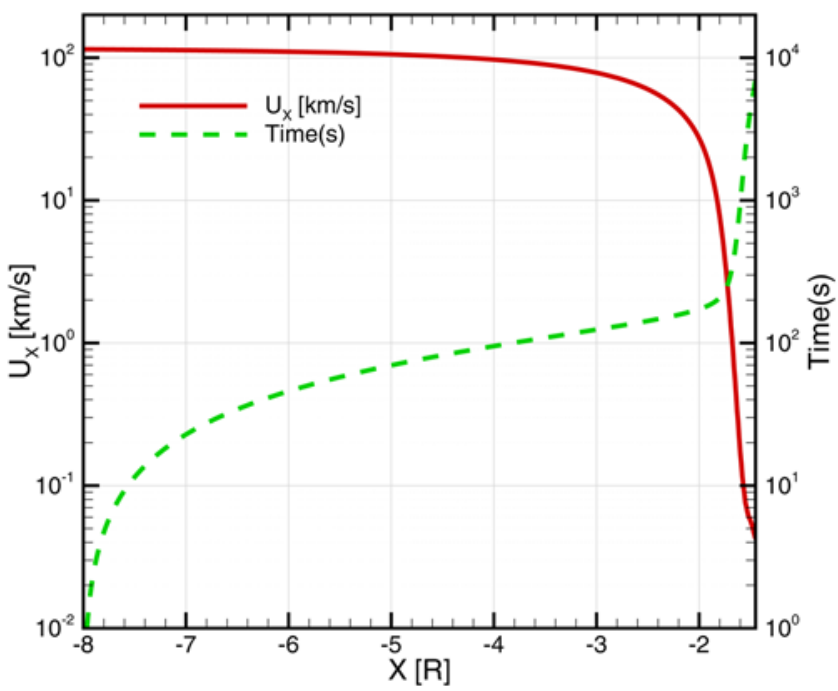

Figure 11. Plasma flow speed and approximate travel time along the ram direction ( $x$ axis) from $-8 R_{T}$ for the steady state case. 
Table 1. Approximate Travel Time From $-8 R_{T}$ to Titan's Upper Ionosphere Along the Ram Direction

\begin{tabular}{cccccc}
\hline Altitude $(\mathrm{km})$ & 2500 & 1600 & 1500 & 1400 & 1200 \\
\hline Travel time & $3 \mathrm{~min}$ & $11 \mathrm{~min}$ & $21 \mathrm{~min}$ & $41 \mathrm{~min}$ & $1 \mathrm{~h} 40 \mathrm{~min}$ \\
\hline
\end{tabular}

was also significantly enhanced. Simulation shows that it could be 2.5 times larger than the normal value during the event.

\section{Discussion and Summary}

[35] The simulation results presented in the paper clearly demonstrated the existence of a fossil field in Titan's ionosphere when the external field changes. The main interaction features of the magnetometer are well reproduced by both the simple current sheet crossing and the more realistic magnetopause crossing cases. The plasma observations are also reproduced reasonably well with the magnetopause crossing case. Simulation results also show that interesting processes happen during the magnetopause crossing, including two pileup regions with opposite magnetic field directions, the formation of a pair of new Alfven wings, and tail disconnection.

[36] The fossil field was trapped in the ionosphere mainly because the convection times in those regions are significantly long. It is also useful to estimate how long the fossil field can stay in the deep ionosphere. Figure 11 shows the plasma flow speed and approximate travel time along the ram direction from $-8 R_{T}$ for the steady state case. As denoted by the red line, the plasma flow speed gradually slows down from the far upstream region. At $2 R_{T}$ upstream, the flow speed is about $25 \mathrm{~km} / \mathrm{s}$, less than $1 / 4$ of the original flow speed. Closer to Titan, the flow speed drops sharply to less than $1 \mathrm{~km} / \mathrm{s}$ and thus takes a significantly longer time for the plasma flow to get to a lower altitude. As listed in Table 1, it takes only $3 \mathrm{~min}$ to get to $2500 \mathrm{~km}$ altitude, but the travel time increases to $1 \mathrm{~h}$ and $40 \mathrm{~min}$ to reach $1200 \mathrm{~km}$. The values listed in Table 1 provide a good reference for the lifetime of the magnetic field near Titan under similar conditions.

[37] However, the travel time also depends on Titan's orbital location and upstream plasma flow condition. Bertucci et al. [2008] pointed out that Titan may have been located in the solar wind for a short duration, about 13 hours before the close encounter of T32. We ran a case with upstream parameters according to the typical solar wind plasma near Saturn (with proton density $=0.1 \mathrm{~cm}^{-3}$, flow speed $U=400 \mathrm{~km} / \mathrm{s}$, plasma temperature $T_{P}=180 \mathrm{eV}$, and $B_{Z}=0.5 \mathrm{nT}$ ) to evaluate the lifetime of Titan's induced magnetic field in the solar wind. In this case, the plasma flow needs only $25 \mathrm{~s}$ from $-8 R_{T}$ to get to the shock location $\left(\sim 4.3 R_{T}\right)$. Inside the shock, the travel time shortly increases to $1.5 \mathrm{~h}$ to the ionopause $(\sim 2400 \mathrm{~km})$. The field below the ionopause is expected to be shielded by the ionosphere. The above case is corresponding to normal solar wind condition; with increased solar wind pressure, the ionopause would move to a lower altitude. In any condition, the lifetime of the induced field was not expected to exceed more than a few hours. Therefore, even though Titan carried some solar wind fossil fields into the magnetosheath for a short time, it is very unlikely that the solar wind fossil fields were still present during the close encounter.

[38] We used a relatively coarse grid $(\sim 60 \mathrm{~km})$ in the ionosphere below $2000 \mathrm{~km}$ for the time-dependent cases (case 2 and case 3 ). Such a grid size is comparable with the scale heights of the main neutrals at any particular locations, which is twice the radial grid size that is used for the steady state case (case 1). As shown in Figure 4, the ion densities as predicted by the high-resolution steady state case fit very well with the LP observation in the ionosphere. The numerical diffusion is expected to be relatively large for the low-resolution cases. As a result, the density dip predicted by the time-dependent cases is not as low as observed near the closest approach. This means that we do want to use high resolution in the ionosphere whenever possible. However, the density profiles among different cases do share a similar trend. In addition, as shown in Figure 3, different resolution does not have a noticeable effect on the calculated magnetic field in the ionosphere. So the resolution used in the time-dependent cases is good enough and should only have limited effect on the results.

[39] Another interesting question is whether the inner boundary would affect the simulation results, especially the estimation of the lifetime of the fossil field in the ionosphere. As mentioned in section 2, we apply a zerogradient boundary condition for both $U$ and $B$. This boundary is consistent with the plasma condition near the inner boundary. Therefore the estimation of lifetime is unlikely to be affected by the boundary conditions of the model. However, we are not sufficiently confident to infer the conditions in Titan's lower atmosphere or on the surface on the basis of model results, since according to Kliore et al. [2008], there is also another significant ionospheric conducting layer in the region of 500-600 km altitude, which is currently neglected in the model.

[40] The density used in the calculation is about 4 times higher than CAPS' recalibrated density. Although the results with lower density have similar trends, they do not fit with the data as well as the high-density cases as shown in the paper. As discussed above, some discrepancy between the data and model could be due to the magnetosheath plasma flow coming in a different direction or at a different speed. However, the flow direction and velocity data are not yet available. It is currently too computationally time consuming to explore the effects of flow direction and different speed without any constraints from observations.

[41] Even the simulated magnetopause crossing case is still much simpler than the real situation. For example, the width of the magnetopause layer is neglected, and the flow condition could be different from that assumed. This will not affect the main conclusions based on the simulated results, though. Because of the significant slow down of the plasma speed in the region close to Titan, the magnetic field in the deep ionosphere could be "fossilized" for hours before it is eroded by the new field. The observations and simulations results of the T32 flyby have important implications for understanding the magnetic field of other Titan flybys. Since the plasma is highly dynamic in Saturn's outer magnetosphere, even though the ambient plasma condition is not changing as dramatically as discussed in the presented flyby, Titan's ionosphere could still record some of those 
changes so that the observed field in the deep ionosphere might have very complicated signatures. The same idea could also apply to Venus and Mars (in the weak crustal field region), which would help us to understand the complicated magnetic signatures in unmagnetized planetary ionospheres.

[42] Acknowledgments. This work was supported by the National Aeronautics and Space Administration through Jet Propulsion Laboratory under contract JPL 1236948. We thank G. R. Lewis and L. K. Gilbert for spectrogram plotting and data analysis software. We acknowledge STFC (UK) for financial support of E.L.S. and NASA/JPL contract 1243218 for financial support of the CAPS investigation. The MHD simulations were preformed on NASA's Columbia High-End Computing systems.

[43] Wolfgang Baumjohann thanks Janet Luhmann and Uwe Motschmann for their assistance in evaluating this manuscript.

\section{References}

Backes, H. (2004), Titan's interaction with the Saturnian magnetospheric plasma, Ph.D. thesis, Univ. of Cologne, Cologne, Germany.

Backes, H., et al. (2005), Titan's magnetic field signature during the first Cassini encounter, Science, 308, 992, doi:10.1126/science.1109763.

Bertucci, C., et al. (2008), The magnetic memory of Titan's ionized atmosphere, Science, 321, 1475, doi:10.1126/science.1159780.

Coates, A. J., et al. (2007), Titan at Saturn's magnetopause: CAPS results from T32, Eos Trans. AGU, 88(52), Fall Meet. Suppl., Abstract P43A-1021.

Cravens, T. E., et al. (2006), Composition of Titan's ionosphere, Geophys. Res. Lett., 33, L07105, doi:10.1029/2005GL025575.

De La Haye, V., et al. (2007), Cassini Ion and Neutral Mass Spectrometer data in Titan's upper atmosphere and exosphere: Observation of a suprathermal corona, J. Geophys. Res., 112, A07309, doi:10.1029/ 2006JA012222.

Dougherty, M. K., et al. (2004), The Cassini magnetic field investigation, Space Sci. Rev., 114, 331, doi:10.1007/s11214-004-1432-2.

Gombosi, T. I., and K. C. Hansen (2005), Saturn's variable magnetosphere, Science, 307, 1224, doi:10.1126/science.1108226.

Gombosi, T. I., K. C. Hansen, and D. L. De Zeeuw (2006), Periodic behavior of Saturn's magnetosphere and Titan's local environment, Eos Trans. $A G U, 87(52)$, Fall Meet. Suppl., Abstract SM31C-02.

Gurnett, D. A., et al. (2004), The Cassini radio and plasma wave investigation, Space Sci. Rev., 114, 395, doi:10.1007/s11214-004-1434-0.

Jia, Y. D., M. R. Combi, K. C. Hansen, T. I. Gombosi, F. J. Crary, and D. T. Young (2008), A 3-D global MHD model for the effect of neutral jets during the Deep Space 1 Comet 19P/Borrelly flyby, Icarus, 196(1), 249-257, doi:10/1016/j.icarus.2008.03.010.

Kallio, E., et al. (2007), Morphology of the magnetic field near Titan: Hybrid model study of the Cassini T9 flyby, Geophys. Res. Lett., 34, L24S09, doi:10.1029/2007GL030827.

Keller, C. N. (1992), One dimensional multispecies magnetohydrodynamic models of the ionosphere of Titan and the inner coma of comet P/Halley, Ph.D. thesis, Univ. of Kansas, Lawrence.

Kliore, A. J., et al. (2008), First results from the Cassini radio occultations of the Titan ionosphere, J. Geophys. Res., 113, A09317, doi:10.1029/ 2007JA012965.

Ledvina, S. A., et al. (2004), Titan's induced magnetosphere, Adv. Space Res., 33, 2092, doi:10.1016/j.asr.2003.07.056.

Ma, Y.-J., A. F. Nagy, T. E. Cravens, I. V. Sokolov, J. Clark, and K. C. Hansen (2004), 3-D Global MHD model prediction of the first close flyby of Titan by Cassini, Geophys. Res. Lett., 31, L22803, doi:10.1029/2004GL021215.
Ma, Y.-J., et al. (2006), Comparisons between MHD model calculations and observations of Cassini flybys of Titan, J. Geophys. Res., 111, A05207, doi:10.1029/2005JA011481.

Ma, Y.-J., et al. (2007), 3D global multi-species Hall-MHD simulation of the Cassini T9 flyby, Geophys. Res. Lett., 34, L24S10, doi:10.1029/ 2007 GL031627.

Modolo, R., and G. M. Chanteur (2008), A global hybrid model for Titan's interaction with the Kronian plasma: Application to the Cassini Ta flyby, J. Geophys. Res., 113, A01317, doi:10.1029/2007JA012453.

Modolo, R., et al. (2007), Plasma environment in the wake of Titan from hybrid simulation: A case study, Geophys. Res. Lett., 34, L24S07, doi:10.1029/2007GL030489.

Nagy, A. F., and T. E. Cravens (1998), Titan's ionosphere: A review, Planet. Space Sci., 46(9-10), 1149.

Neubauer, F. M., et al. (2006), Titan's near magnetotail from magnetic field and electron plasma observations and modeling: Cassini flybys TA, TB, and T3, J. Geophys. Res., 111, A10220, doi:10.1029/2006JA011676.

Powell, K. G., P. L. Roe, T. J. Linde, T. I. Gombosi, and D. L. DeZeeuw (1999), A solution-adaptive upwind scheme for ideal magnetohydrodynamics, J. Comput. Phys., 154, 284, doi:10.1006/jcph.1999.6299.

Simon, S., U. Motschmann, G. Kleindienst, K.-H. Glassmeier, C. Bertucci, and M. K. Dougherty (2008a), Titan's magnetic field signature during the Cassini T34 flyby: Comparison between hybrid simulations and MAG data, Geophys. Res. Lett., 35, L04107, doi:10.1029/2007GL033056.

Simon, S., et al. (2008b), Influence of non-stationary electromagnetic field conditions on ion pick-up at Titan: 3-D multispecies hybrid simulations, Ann. Geophys., 26, 599.

Smith, G. R., et al. (1982), Titan's upper atmosphere: Composition and temperature from the EUV solar occultation results, J. Geophys. Res., 87, 1351, doi:10.1029/JA087iA03p01351.

Snowden, D., R. Winglee, C. Bertucci, and M. Dougherty (2007), Threedimensional multifluid simulation of the plasma interaction at Titan, J. Geophys. Res., 112, A12221, doi:10.1029/2007JA012393.

Wahlund, J.-E., et al. (2005), Cassini measurements of cold plasma in the ionosphere of Titan, Science, 308, 986, doi:10.1126/science.1109807.

Waite, J. H., et al. (2005), Ion Neutral Mass Spectrometer results from the first flyby of Titan, Science, 308(5724), 982-986, doi:10.1126/ science. 1110652 .

Young, D. T., et al. (2004), The Cassini Plasma Spectrometer, Space Sci. Rev., 114, 1, doi:10.1007/s11214-004-1406-4.

C. Bertucci and M. K. Dougherty, Blackett Laboratory, Space and Atmospheric Physics Group, Imperial College London, Prince Consort Road, London SW7 2AZ, UK.

A. J. Coates and A. Wellbrock, Mullard Space Science Laboratory, Department of Space and Climate Physics, University College London, London RH5 6NT, UK.

F. J. Crary, Southwest Research Institute, 6220 Culebra Road, San Antonio, TX 78228, USA.

T. E. Cravens, Department of Physics and Astronomy, University of Kansas, 1251 Wescoe Hall Drive, Lawrence, KS 66045, USA.

P. Garnier and J.-E. Wahlund, Swedish Institute of Space Physics, Box 537, Uppsala SE-75121, Sweden.

Y. J. Ma and C. T. Russell, Institute of Geophysics and Planetary Physics, University of California Los Angeles, 3845 Slichter Hall, 603 Charles E. Young Drive, E, Los Angeles, CA 90095, USA. (yingjuan@igpp.ucla.edu) A. F. Nagy and G. Toth, Department of Atmospheric, Oceanic and Space Sciences, University of Michigan, 2455 Hayward Street, Ann Arbor, MI 48109, USA.

F. M. Neubauer, Institute of Geophysics and Meteorology, University of Cologne, Zülpicher Strasse 49, D-50674 Cologne, Germany. 